\title{
Impact of Self-Coiling Catheters for Continuous Popliteal Sciatic Block on Postoperative Pain Level and Dislocation Rate: A Randomized Controlled Trial
}

\section{Rosa Nickl ( $\sim$ rosa.nickl@ukdd.de )}

University Hospital Carl Gustav Carus Dresden, Technische Universität Dresden

Oliver Vicent

University Hospital Carl Gustav Carus Dresden, Technische Universität Dresden

Thomas Müller

University Hospital Carl Gustav Carus Dresden, Technische Universität Dresden

Anne Osmers

University Hospital Carl Gustav Carus Dresden, Technische Universität Dresden

Konrad Schubert

Technische Universität Dresden

Thea Koch

University Hospital Carl Gustav Carus Dresden, Technische Universität Dresden

Torsten Richter

University Hospital Carl Gustav Carus Dresden, Technische Universität Dresden

\section{Research Article}

Keywords: perineural catheter, popliteal sciatic block, self-coiling catheter, dislocation, ultrasound- guided re-gional anesthesia

Posted Date: October 26th, 2021

DOl: https://doi.org/10.21203/rs.3.rs-959997/v1

License: (c) (1) This work is licensed under a Creative Commons Attribution 4.0 International License.

Read Full License 


\section{Abstract}

Background: Catheter dislocation within the tissue is a challenge in continuous regional anesthesia. A novel self-coiling catheter design has been available providing lower dislocation rate in a cadaver model. So far it hasn't been demonstrated whether the self-coiling catheter offers any remarkable advantages for continuous peripheral regional anesthesia in vivo.

Methods: After ethics committee approval 140 patients undergoing elective distal lower limb surgery were enrolled in this prospective randomized controlled trial. Preoperatively, patients were randomly assigned and received either the conventional $(n=70)$ or self-coiling catheter $(n=70)$ for ultrasound-guided popliteal sciatic nerve block in short axis view and in-plane approach from lateral. The primary endpoint was pain intensity after surgery and on the following days. Secondary outcomes investigated were dislocation rate in situ, externally visible catheter movement, opioid consumption as well as leakage at the puncture site.

Results: All catheters were successfully inserted. The study population of self-coiling catheters had significantly lower mean numeric rating scale values than the reference cohort on the first $(p=0.01)$ and second postoperative days $(p<0.01)$. Sonographic evaluation has shown, 42 standard catheters $(60 \%)$ and 10 self-coiling catheters ( $14.3 \%)$ were dislocated in situ within the first three postoperative days. The externally visible movement of the catheters at insertion site did not differ significantly and was on average less than $0.5 \mathrm{~cm}$ on the third day. The amount of opioids administered was significantly lower in the self-coiling catheter group on the day of surgery and on the second and third postoperative days $(p=0.04, p=0.03$ and $p=0.04$, respectively).

Conclusion: The self-coiling catheter offers a superior postoperative pain control and a lower dislocation rate within the tissue for popliteal sciatic nerve blockade compared to a conventional catheter. Further trials in large patient cohorts are warranted to investigate potential beneficial effects of self-coiling catheters for other localisations and other application techniques.

Trial registration: The trial was registered at German Clinical Trials Register (DRKS) on 08/04/2020 (DRKS00020938, retrospectively registered).

\section{Background}

Continuous nerve blocks play an integral role in modern multimodal analgesia concepts [1]. Regional anesthesia is essential in orthopaedic and trauma surgery on the lower limb [2]. For both, the lower and upper extremities, randomized controlled trials have shown significant pain reduction by means of continuous regional anesthesia after surgery $[3,4]$. This also results in less chronic pain, a lower opioid requirement and associated side effects such as nausea, vomiting, constipation and fatigue $[3,5]$. However, in some studies analgesic advantages of continuous regional anesthesia fade after 24- $48 \mathrm{~h} \mathrm{[6}$, 7]. The secondary failure rate of indwelling regional anesthesia catheters has been reported in literature up to $40 \%[8,9]$. Aside from initial misplacement secondary catheter dislocation during the postoperative course might be considered one possible explanation for the worsened efficacy of continuous regional 
anesthesia [10]. Secondary dislocation is commonly defined as outwardly visible displacement or sliding out of the catheter, partly accidently by the patient himself $[11,12]$. However, regarding dislocations, the rare event of external dislodgement at the insertion site has to be distinguished from internal catheter tip migration away from target structure or nerve surrounding compartment due to active and passive movement of adjacent muscles. The latter problem might be underestimated since pain scores, opioid consumption, sensory block distribution and patient's satisfaction serve only unreliable surrogate measures for correct catheter position. Investigation of internal dislocations by direct visualization of the catheter tip or better by imaging of fluid spread referred to the nerve has been addressed only in a few studies with limited number of patients $[10,13,14]$. Two more studies have investigated dislocation rate in situ, either solely in healthy volunteers [15] or in human cadavers [16]. Stiff catheters placement using the most popular short axis (SAX) / in-plane (IP) approach might bear an increased risk of internal dislocations $[10,16]$ since the catheter mostly can be placed only a short distance beyond the needle tip to avoid bypassing the nerve [17].

For a new catheter design it has been shown a very low risk of initial misplacement in cadavers for paravertebral blockades as well as sciatic nerve blockades $[18,19]$ due to a $2.5 \mathrm{~cm}$ long self-coiling soft end of the catheter remaining close to the nerve. However, so far, no studies have investigated if selfcoiling catheters are also more resistant to secondary dislocations with consecutive improvement of pain management in surgical patients.

Therefore, we compared the self-coiling catheter with regular straight catheters for continuous popliteal sciatic blockade regarding efficacy and position change within and outside the tissue.

\section{Methods}

\section{Study design}

This study is a prospective, randomized controlled, single-centre trial in a clinically interventional design.

\section{Ethics}

Positive ethic votum was approved by the Institutional Review Board of the Technische Universität Dresden (EK150042016). Written informed consent was obtained from all patients. This study is registered at the German Clinical Trials Register (DRKS00020938) and is reported according to the CONSORT guidelines 2010 [20].

\section{Patients and randomisation}

140 adult patients scheduled for continuous regional anesthesia with popliteal sciatic catheter as part of elective major surgery on the ankle or foot in the University Hospital Carl Gustav Carus at the Technische Universität Dresden, were enrolled between 09/2016 and 12/2017 for this trial. The applied inclusion and exclusion criteria are summarized in Table 1. Randomisation was carried out immediately before catheter insertion by means of sequentially numbered sealed opaque envelopes, containing the study number and 
corresponding reference to the respective group. Patients were assigned to two groups: an interventional group with the application of the self-coiling catheter, SCC, (SonoLong Curl Echo $20 \mathrm{G} 100 \mathrm{~mm}$, Pajunk medical products, Geisingen, Germany) and a control group receiving the regular straight catheter, RSC, (SonoLong Sono $20 \mathrm{G} 100$ mm, Pajunk medical products, Geisingen, Germany) for popliteal sciatic block (figure 1). Surgeons, nurses, patients and members of the acute pain service and investigators apart from the anesthetists inserting the catheter were blinded to the study group. Investigators collecting data were not blinded to groups.

Table 1

Inclusion and exclusion criteria

Inclusion criteria

- age between 18 and 75 years

- indication for the application of a distal sciatic catheter within the scope of an elective surgical procedure

- patient consent

\section{Exclusion criteria}

- patient suffering from chronic pain before surgery

- polyneuropathy or ipsilateral neuropathy, involving the lower limb

- refusal of regional anesthesia

- patient not legally competent

- intolerance or allergy to ropivacaine or oxy-codone

- neuromuscular diseases

- $\mathrm{BMI}>35 \mathrm{~kg} / \mathrm{m}^{2}$

- pre-existing opioid medication

\section{Application of regional anesthesia}

Patients were placed in supine position with the relevant leg elevated. After generous skin disinfection (ChloraPrep, Becton, Dickinson and Company, Franklin Lakes, USA) the area was covered with a sterile fenestrated sheet. The transducer was draped with a sterile ultrasound probe cover (CIV-Flex ${ }^{\circledR}$ Transducer Covers, Civco Medical Solutions, Kalona, lowa, USA).The sciatic nerve was visualized from the posterior thigh in short axis view (SAX) at the level of the popliteal nerve bifurcation with a 4-12 $\mathrm{MHz}$ linear probe (L12-4) of a Philips Sparq or Philips Affiniti 70G ultrasound system (Philips Healthcare, Andover, Massachusetts, USA) or 5- $13 \mathrm{MHz}$ linear probe (12L-RS) of a GE Logiq e ultrasound system (GE Healthcare, Milwaukee, Wisconsin, USA). Nerve bifurcation was defined as the most proximal point where the tibial and common fibular nerve clearly started to detach. After skin infiltration with 2-4 $\mathrm{ml}$ prilocaine 1 $\%$, an $18 \mathrm{G}$ Tuohy needle was advanced using an in-plane technique from lateral until the needle tip was located within the paraneural sheath. The designation of the sheaths of the sciatic nerve is referred to the previous publication of Andersen and colleagues [21]. Special care was taken not to touch the nerve or even puncture the epineurium. An initial bolus of $20 \mathrm{ml}$ ropivacaine $0.5 \%$ (Naropin $10 \mathrm{mg} / \mathrm{ml}$, Astra Zeneca, London, UK) was applied directly via the injection line connected to the Tuohy puncture cannula 
under direct sonographic control. The desired spread of local anesthetic was circularly around both components of sciatic nerve within the paraneural sheath. If diffuse spreading into the surrounding tissue occurred, the cannula position was corrected. Afterwards, the previously randomized catheter was placed adjacent to the sciatic nerve within the paraneural sheath through the cannula. According to manufacturer instructions SCC was advanced approximately $2.5-3 \mathrm{~cm}$ beyond the needle tip to facilitate the coiling up of the distal catheter end. RSC was inserted approximately $3-4 \mathrm{~cm}$ past the needle tip. Subsequently proper catheter position was sonographically confirmed by observing the spread of a $2 \mathrm{ml}$ bolus of saline injected via the catheter within the paraneural sheath. In case of spread outside of paraneural sheath catheter was retracted under real-time sonographic guidance until the injected saline bolus was reliably distributed around the nerves. Thereafter the catheter was connected to the associated bacterial filter and fixed with sterile wound suture strips (Omnistrip ${ }^{\circledR}$, Fa. Paul Hartmann AG, Heidenheim, Germany). Finally, a sterile foil dressing (IV3000 10x12 cm, Smith \& Nephew Medical Ltd., London, UK) was used to provide fixation of the catheter additionally. All catheters were placed as a part of the clinical anesthesia routine by a total of four senior anesthesiologists with substantial experience in performing ultrasound-guided popliteal sciatic nerve block. The success of the sciatic blockade was evaluated in all patients by testing warm-cold differentiation in the innervation area.

\section{Additional anesthetic procedures and hemodynamic monitoring}

The time course of proceeded interventions were summarized in figure 2. After arriving in the operating area, peripheral venous access with an infusion of a balanced crystalloid solution, a 3- or 5-lead ECG monitoring including ST-segment analysis, a pulsoxymetry and non-invasive blood pressure measurement were established. Hemodynamic data were continuously recorded using a Philips Intellivue MP 70 (Philips Medicine Systems GmbH, Hamburg, Germany). If surgery involved the medial area of the lower leg, ankle or foot, an additional ultrasound guided saphenous nerve block was performed with 10 $\mathrm{ml}$ ropivacaine $0.5 \%$ (Naropin $10 \mathrm{mg} / \mathrm{ml}$, Astra Zeneca, London, UK) via an $80 \mathrm{~mm} 22$ G Sonoplex cannula (Pajunk medical products, Geisingen, Germany) at femoral triangle by SAX view and in-plane approach. Further anesthesia procedures were based on the individual risk profile, patient's comfort request and needs due to planned surgery procedures like tourniquet at the thigh. Independent of the randomization, in addition to the continuous peripheral sciatic blockade the following procedures were used: anesthesia standby, sedation, femoral and obturator nerve block and spinal or general anesthesia. For spinal anesthesia 2 to $2.4 \mathrm{ml}$ of hyperbaric bupivacaine $0.5 \%$ (Bucain hyperbar $5 \mathrm{mg} / \mathrm{ml}$, PUREN Pharma GmbH \& Co. KG, Munich, Germany) with $10 \mu \mathrm{g}$ fentanyl via a $25 \mathrm{G}$ Sprotte cannula (Pajunk medical products, Geisingen, Germany) was used. Sedation was applied by use of propofol $20 \mathrm{mg} / \mathrm{ml}$ (Fresenius Kabi Deutschland GmbH, Bad Homburg, Germany) with a rate of $1-2 \mathrm{mg} / \mathrm{kg} / \mathrm{h}$. General anesthesia was induced and maintained with propofol (Propofol $1 \%$ and Propofol $2 \%$, Fresenius Kabi Deutschland GmbH, Bad Homburg, Germany) and sufentanile (Sufentanil-hameln $5 \mu \mathrm{g} / \mathrm{ml}$, hameln pharmaceuticals gmbh, Hameln, Germany). The airway was secured by laryngeal mask (Ambu® AuraGain $^{\mathrm{TM}}$, Ambu GmbH, Bad Nauheim, Germany). 


\section{Postoperative procedure and outcome parameter assessment}

After surgery, the patients were observed under cardiovascular monitoring in post anesthesia care unit, as appropriate. Afterwards they were transferred to general ward. Postoperatively, both groups received a continuous application of ropivacaine $0.2 \%$ (Naropin $2 \mathrm{mg} / \mathrm{ml}$, Astra Zeneca, London UK) at an initial rate of $6 \mathrm{ml} / \mathrm{h}$. According to a multimodal pain management concept, all patients received oral ibuprofen $600 \mathrm{mg}$ as basic analgesia every eight hours. In case of contraindications for ibuprofen, novamine sulfone ( $1 \mathrm{~g}$ every six hours) was administered as an alternative per os or intravenously. A pre-existing basic analgesia with novamine sulfone and ibuprofen, in the above-mentioned dosages, was maintained postoperatively. The patients were regularly visited by the acute pain service from the first post-operative day (POD) twice a day. Pain service visit included surveillance of infection signs, leakage and external dislocation at insertion site. In order to record the subjective pain level of the patient twice a day the numerical rating scale (NRS 0-10) was used as a semi-quantitative method. The first interview was conducted two hours after the end of the operation. The NRS, the spread of the sciatic blockage and any adverse events were recorded. For rescue pain (NRS>3) either, a manual bolus of $10 \mathrm{ml}$ ropivacaine $0,3 \%$ (Naropin, Astra Zeneca, London, UK) could be applicated by acute pain service staff or patients received oxycodone $10 \mathrm{mg}$ (Oxygesic akut $10 \mathrm{mg}$, Mundipharma GmbH, Limburg, Germany) per os by ward nurses. The total daily requirement of ropivacaine and total oxycodone consumption was recorded.

The position of indwelling catheters were revealed immediately after surgery at the PACU and daily on the first, second and third POD. For this purpose, distribution of a saline a bolus of 2- $8 \mathrm{ml}$ via the catheter was observed under sonographic view with respect to the nerve and the adjacent paraneural sheath. Corresponding images were digitally stored and the detected position was documented. Three different positions of the catheter in relation to the nerve were categorized as depicted in figure 3 . In case of catheter dislocation, no further US-guided examinations were performed afterwards.

\section{Safety thresholds}

Participation in the trial was terminated at any time, if one of the following dropout criteria was fulfilled: request of the patient, allergic reaction to ropivacaine, novamine sulfone or ibuprofen, necessity of second operation during the follow up period.

\section{Primary and secondary outcomes}

The primary outcome was the individual postoperative pain intensity, assessed with the numerical rating scale (NRS) after surgery on the lower leg or foot in the first 72 hours. The patient classified the pain on a scale from 0 to 10 . The following issues were identified as secondary outcomes: additional need for systemic opioids, rate of secondary dislocations within the tissue and dislocations and leakage at the injection site.

\section{Statistical analysis}


The sample size was calculated as followed. To demonstrate a clinically relevant difference of 1 level on an NRS of 0 to 10, a minimum of 64 patients were enrolled with a standard deviation of 2, a significance level of 0.05 and a power of $80 \%$. A drop-out rate of $8 \%$ was expected. Therefore, 70 patients per group were included. In accordance with the case number planning, the evaluation was carried out with an unpaired two-sided Student's t-test at $5 \%$ level, assuming equality of variance. The distribution of continuous parameters was described by using of mean value and standard deviation (normally distributed) or by medians and quartiles. Categorical parameters were described by their absolute and relative frequencies, and differences between groups were examined using the Chi- square test according to Pearson or the exact test according to Fisher. Two-group comparisons of metric scaled variables were performed by independent two-sided t-tests after using Levene test checking equality of variances. To analyse the influence of possible covariates of respective baseline data, a covariance analysis was calculated to adjust for possible group differences for confounders. To correlate nominal and metric parameters, eta correlation coefficient was calculated. Statistical significance was considered at twosided $\mathrm{p}<0.05$.

All calculations and graphs were performed and computed using SPSS (IBM SPSS Statistics Vers. 25, IBM Deutschland GmbH, Ehningen, Germany).

\section{Results}

From 09/2016 to 12/2017 1021 patients undergoing elective ankle or foot surgery were screened and 140 patients were enrolled in this study (figure 4). Both groups were comparable regarding baseline characteristics as depicted in Table 2. The study was closed after enrolment of the planned 140 patients. 
Table 2

Baseline characteristics.

\begin{tabular}{|c|c|c|}
\hline & RSC Group ( $n=70)$ & SCC Group $(n=70)$ \\
\hline Age [years] & $50 \pm 14$ & $50 \pm 13$ \\
\hline \multicolumn{3}{|l|}{ Gender [no/\%] } \\
\hline Female & $37(53)$ & $37(53)$ \\
\hline Male & $33(47)$ & $33(47)$ \\
\hline Body height [cm] & $171.7 \pm 9,0$ & $173.1 \pm 10.2$ \\
\hline Body weight [kg] & $76.5 \pm 13.7$ & $79.3 \pm 16.5$ \\
\hline BMI $\left[\mathrm{kg} / \mathrm{m}^{2}\right]$ & $25.9 \pm 3.7$ & $26.3 \pm 4.3$ \\
\hline \multicolumn{3}{|l|}{ ASA [no./\%] } \\
\hline I & $38(54)$ & $35(50)$ \\
\hline II & $27(39)$ & $33(47)$ \\
\hline III & $5(7)$ & $2(3)$ \\
\hline \multicolumn{3}{|l|}{ Surgical area } \\
\hline Upper ankle joint & $35(50)$ & $41(59)$ \\
\hline Calcaneus & $8(11)$ & $14(20)$ \\
\hline Hallux & $10(14)$ & $6(9)$ \\
\hline Talus & $6(9)$ & $0(0)$ \\
\hline Metatarsal & $10(14.3)$ & $7(10)$ \\
\hline $\begin{array}{l}\text { Tendons/syndesmosis } \\
\text { of the ankle }\end{array}$ & $1(1)$ & $2(3)$ \\
\hline $\begin{array}{l}\text { Values are given as abs } \\
\text { ASA American Society o } \\
\text { catheter, } S C C \text { self-coiling }\end{array}$ & $\begin{array}{l}\text { Dercentage) or mean } \\
\text { gy physical status, } E\end{array}$ & $\begin{array}{l}\text { deviation), as appropriate. } \\
\text { ss index, RSC regular straight }\end{array}$ \\
\hline
\end{tabular}

\section{Catheter placement and additional anesthetic procedures}

Sonographic identification of the nerve and bifurcation was successfully possible in all patients. The mean performance time acquired for catheter placement was $12.9 \pm 4.3 \mathrm{~min}(p=0.97)$ in both study groups. The catheter placement was successful in all patients indicated by the correct local anesthetic distribution within the paraneural sheath of the sciatic nerve and complete loss of sensory and motoric function in the supplied nerve territory. As shown in Table 3, there was no statistic significant difference in 
duration of surgery and initial amount of administered ropivacaine. No difference between groups were detected regarding the number of additional saphenous nerve blocks and other additional anesthetic procedures as well as to the usage of postoperative basic analgesic medication. 
Table 3

Additional anesthetic procedures, duration of catheter placement and surgery, postoperative basic analgesic medication, ropivacaine consumption and leakage.

\begin{tabular}{|c|c|c|c|}
\hline & RSC Group & SCC Group & p Value \\
\hline Saphenous nerve block & $64(91.4)$ & $56(80)$ & 0.05 \\
\hline Additional anesthetic procedure [no/\%] & & & 0.17 \\
\hline General anesthesia & $53(75.7)$ & $60(85.7)$ & \\
\hline Spinal anesthesia & $10(14.3)$ & $6(8.6)$ & \\
\hline Femoral nerve and obturator nerve block & $0(0)$ & $2(2.9)$ & \\
\hline Analgosedation & $6(8.6)$ & $2(2.9)$ & \\
\hline none & $1(1.4)$ & $0(0)$ & \\
\hline Duration of catheter placement [min] & $12.9 \pm 4.3$ & $12.9 \pm 4.3$ & 0.97 \\
\hline Duration of surgery [min] & $94.4 \pm 41.0$ & $96.2 \pm 39.4$ & 0.79 \\
\hline Postop. analgesic medication [no/\%] & & & 0.2 \\
\hline Ibuprofen & $46(65.7)$ & $55(78.6)$ & \\
\hline Novamine sulfone & $19(27.1)$ & $13(18.6)$ & \\
\hline Ibuprofen+ Novamine sulfone & $5(7.1)$ & $2(2.9)$ & \\
\hline \multicolumn{4}{|l|}{ Ropivacaine consumption [mg/ 24h] } \\
\hline POD 0 & $283.8 \pm 34.7$ & $288.7 \pm 10.0$ & 0.26 \\
\hline POD 1 & $264 \pm 112.1$ & $233 \pm 101.5$ & 0.09 \\
\hline POD 2 & $108 \pm 124.9$ & $127.5 \pm 116.7$ & 0.38 \\
\hline POD 3 & $23 \pm 84.4$ & $52.1 \pm 93.4$ & 0.22 \\
\hline \multicolumn{4}{|l|}{ Leakage [no/\%] } \\
\hline POD 0 & $8(11.4)$ & $6(8.6)$ & 0.83 \\
\hline POD 1 & $22(31.4)$ & $21(30)$ & 0.84 \\
\hline POD 2 & $21(30)$ & $22(31.4)$ & 0.6 \\
\hline POD 3 & $3(4.3)$ & $9(12.9)$ & 0.07 \\
\hline $\begin{array}{l}\text { Values are given as absolute number (per } \\
\text { Difference between groups were tested wi } \\
\text { statistical significance considered at } p<0 \text {. } \\
\text { SCC self-coiling catheter. }\end{array}$ & $\begin{array}{l}r \text { mean }( \pm \text { stan } \\
\text { are test or two } \\
\text { stoperative da }\end{array}$ & $\begin{array}{l}\text { deviation), as } \\
\text { d Student's t-te } \\
\text { SC regular straic }\end{array}$ & $\begin{array}{l}\text { ppriate. } \\
\text { h } \\
\text { theter, }\end{array}$ \\
\hline
\end{tabular}




\section{Primary outcome parameter- postoperative pain level}

The pain levels recorded daily over the observation period are depicted in figure 5 . The NRS scores did not differ significantly on the day of surgery $(p=0.69)$. On POD 1 and POD 2 , patients of SCC group showed significantly less pain than those in the RSC group. There was no difference in pain intensity between both study populations on POD 3.

\section{Secondary outcome parameters}

\section{Opioid consumption}

The values of oxycodone consumption determined showed a wide spread and are summarized in Table 4. All mean values of the oxycodone consumption are lower with the self-coiling catheter than with the conventional catheter. Significant differences between both groups were found on the day of surgery $(p=0.04)$, POD $2(p=0.03)$ and POD $3(p=0.04)$. For POD $1(p=0.19)$ a tendency in lower oxycodone consumption have been observed.

Table 4

Oxycodone consumption of both study groups during study period.

\begin{tabular}{|c|c|c|c|}
\hline & RSC Group & SCC Group & p Value \\
\hline \multicolumn{4}{|c|}{ Daily Oxycodone consumption (mg) } \\
\hline POD 0 & $1.29 \pm 4.45$ & $0.14 \pm 1.19$ & $0.04 *$ \\
\hline POD 1 & $15.57 \pm 19.16$ & $11.71 \pm 15.32$ & 0.19 \\
\hline POD 2 & $12.50 \pm 14.90$ & $7.57 \pm 11.60$ & $0.03 *$ \\
\hline POD 3 & $7.94 \pm 8.64$ & $4.92 \pm 7.88$ & $0.04^{*}$ \\
\hline
\end{tabular}

\section{Dislocation}

The spread of fluid could be detected in all sonographic examinations, which were performed throughout the study. Dislocation rates are shown in figure 6 . The majority of dislocations were found already after the surgical procedure and patient transfer to PACU. However, the dislocation of catheter was significant lower in the SCC group. (RSC $n=19$ vs. SCC $n=1, p<0.01$ ). Over the entire observation period, the SCCcohort catheters dislocated less frequently than in the RSC group ( $p<0.01$ for POD 1 \& 2, $p=0.01$ for POD 3 , respectively). The cumulative dislocation rate was $60 \%(n=42)$ in RSC group and $14.3 \%(n=10)$ in SCC group. For the first and second postoperative day, a significant influence of dislocation on the mean indicated NRS value could be demonstrated (POD $1 p<0.01$; POD $2 p<0.01$ ) as shown in figure 7.

\section{Catheter insertion depth}


The puncture depth of both catheter groups did not differ significantly $(p=0.169)$ and averaged 5.8 (RSC) and $6.0 \mathrm{~cm}$ (SCC). However, the distance between the puncture depth and the final skin level of the catheters differed due to catheter design. The self-coiling catheters were placed at a mean of $2.4 \mathrm{~cm}$ above the final needle tip position. For the regular catheter, this value was $1.6 \mathrm{~cm}(p<0.01)$. No catheter in either group with an insertion depth greater than/equal to $3.5 \mathrm{~cm}$ dislocated in this study. The eta coefficient was $0.380(p<0.01)$, resulting in a moderate correlation. Hence, $14.4 \%$ of the dislocation variance can be explained by the insertion depth.

\section{External movement at the insertion site}

The outwardly visible movement of the pain catheters was documented daily. In contrast to the dislocation within the tissue, no significant difference between both groups could be detected over the entire observation period regarding the externally visible position at the insertion site (POD $0, p=0.86 ; P O D$ $1, p=0.39 ;$ POD 2, $p=0.65)$. In total, $7(10 \%)$ of the self- coiling catheters and $9(12.9 \%)$ of the regular straight catheters slipped back at least $1 \mathrm{~cm}$ or further $(p=0.6)$. We could not observe any differences between correctly positioned and dislocated catheters regarding the outward movement of the catheters.

\section{Leakage}

Overall, leakage of the puncture sites was most frequently detected on the first and second postoperative day. We observed no significant differences in characteristics between the SCC and RSC group (POD 0, $p=0.83 ;$ POD 1, $p=0.84 ;$ POD 2, $p=0.6 ;$ POD 3, $p=0.07$ ) as seen in Table 3 .

\section{Complications due to perineural catheter}

Eight patients (5.7\%) experienced minor local inflammation at the insertion site, which was utterly subsiding after removal in the further course of the trial. Because of catheter occlusion, one catheter in each study group $(0.7 \%)$ could no longer be used from the first and second postoperative day. One patient in the RSC group complained of a metallic taste and nausea after increasing the infusion rate of ropivacaine from 6 to $8 \mathrm{ml} / \mathrm{h}$ Ropivacaine $0.2 \%$ and administration of a bolus. The catheter was then removed immediately and all symptoms were completely regressed within a few hours. No neurologic deficit occurred during the observation period.

\section{Discussion}

In this investigator-initiated randomized controlled trial it could be demonstrated that the use of a selfcoiling catheter compared with a regular straight catheter for continuous popliteal sciatic block was more effective in terms of pain management and dislocation rate within the tissue. The latter appeared without visible external changes measured by leakage and external dislodgement of the catheter at the insertion site. The higher stability of the self-coiling catheter in its original positioning may result in lower pain levels on the first and second POD and a reduced opioid consumption compared to patients in the RSC group. To our knowledge this is the first trial investigating impact of different catheter characteristics on 
secondary dislocations and advantages of a self-coiling catheter for peripheral nerve block in clinical practice.

\section{Pain intensity}

Dislocation rate on the day of operation had no impact on pain levels because initial bolus of ropivacaine $0.5 \%$ caused a long-lasting blockage of pain perception in both groups. Long acting ropivacaine provides nerve block duration of up to 18 hours, depending on dose and proximity of applied LA to the nerve. Christiansen et al. described a mean duration of action for distal sciatic blocks of more than 13 hours with a lower dose of $60 \mathrm{mg}$ ropivacaine [22]. In this study $100 \mathrm{mg}$ were administered. Various other studies have reported similar results. Several investigators compared a single popliteal block of the sciatic nerve with a continuous application of local anesthetic via catheter and determined no significant differences in pain intensity on the day of surgery for both groups [23-25]. The effect of the initially performed nerve block was effective in both groups and thus did not result in NRS-differences.

The significance of the lower pain level of the SCC group on the first and second POD showed a measurable difference of 0.7 points on NRS to the control group. Even if this difference seems to be small, it can become relevant for the individual patient. The reason for the higher pain scores in the RSC group on POD 1 and 2 may be related to a higher dislocation rate outward of the fascial space of the sciatic nerve. Thus, a lower effective dose of continuous infused ropivacaine could act on the sciatic nerve. In contrast, the lack of difference on POD 3 might be explained by spontaneous pain relief in the late postoperative period.

With the diminishing effect of the initial bolus of ropivacaine $0.5 \%$ on the first postoperative day, the average pain level of all participants was highest compared to the other measure points. This might be caused by dislocations of indwelling catheters, insufficiency of lower continuous ropivacaine dose and pain perception in the regions not supplied by sciatic nerve when single shot of supplemental regional anesthesia had worn off. The latter issue is relevant if medial parts of the foot were involved in surgery and saphenous or femoral nerve block was established [26]. Pain perception in sensory territory of the saphenous nerve might have contributed to levelling pain intensity scores and scattering intergroup differences regarding perineural sciatic nerve catheter performance. Using an additional catheter for the saphenous nerve might have decreased pain scores and additional opioid use. However, reports about impact of the continuous or prolonged saphenous nerve block on pain after ankle surgery are conflicting. Whereas Fisker et al could not find advantage of continuous saphenous nerve block compared to single shot [27]. Jarell et al. showed lower pain scores and less opioid need with continuous block of saphenous nerve after ankle surgery [28]. Another investigator reported improved postoperative pain management with prolonged saphenous nerve block by additional perineural dexamethasone [29].

Surgery of the distal lower extremity or the foot has shown to be one of the most painful surgical interventions. In almost 71,000 patients with 179 different operations in all areas of the body, the calcaneus operation was revealed to be the most painful operation with a mean postoperative NRS of 
6.68 on the first postoperative day. Other operations on the foot, forefoot or ankle were among the most painful fifteen operations with a mean NRS of at least 6 on the first postoperative day [2]. This underlines the importance of continuous regional anesthesia of the ankle and foot.

In our study almost, all patients were free of pain on POD 0. In the further course, all mean NRS values in both cohorts remained at all examination times below 3.5 but only patients with self-coiling catheters performed with mean NRS 2.7 even lower than the commonly accepted intervention threshold of NRS 3 .

The absence of ultrasound-guidance and confirmation of proper catheter location, differently used local anesthetics and additional analgetic drugs, the heterogeneity of surgical procedures and the lack of information about mobilization strategies, complicate comparability with other studies. However, most studies show maximum pain intensity on POD 1 [23-25, 30-32]. Similar to our results, usually pain level decreases day by day in the further course. The pain intensity of patients with continuous sciatic nerve block varied between 0 and 4 on POD 1. Our results are in this range. Many of the studies mentioned above had an outpatient setting which may be another reason for the fluctuation range in reported pain intensity. Patients were discharged with the peripheral nerve catheters on the day of surgery and received defined amounts of analgetics as well as instructions for mobilisation. Previous surgery, the duration and length of stay in hospital varied greatly or were not specified. The indicated operation times were very heterogeneous and ranged from $32 \mathrm{~min}$ [24] to $110 \mathrm{~min}$ [33]. That may have an impact on surgical trauma and consequently on pain intensity. The evaluation of pain and other criteria in the following days was conducted by telephone. As a result, it could not be clearly elucidated whether patients' statements are reliable and how mobilisation was carried out as additional pain stimulus.

\section{Oxycodone consumption}

Patients in the self-coiling catheter group had a lower need for oxycodone in the first three days after surgery. While the lower consumption of oxycodone in the SCC group differed by $1.1 \mathrm{mg}$ from the RSC group on the day of surgery, already on POD 1 the consumption was $3.9 \mathrm{mg}$ lower. This difference in lower consumption in the SCC group reached significant levels at the day of surgery, POD $2(4.9 \mathrm{mg})$ and POD 3 ( $3 \mathrm{mg}$ ), with an overall decrease in the need for additional opioids already from the second postoperative day on. Interestingly, the significant elevated pain intensity in the RSC group did not reach the level to create a difference for opioid consumption on POD 1.

Two facts might have influence accuracy of discrimination. First, we used a single prefixed dose of oxycodone $10 \mathrm{mg}$ as rescue pain medication obeying our institutional multimodal pain concept. Therefore, discriminatory power regarding additional opioid need was low. Assumably, with lower opioid bolus provided by patient controlled intravenous analgesia we could have traced the opioid requirement more precisely. Second, over 50 \% of patients in both groups had surgery that involved also innervation territory of saphenous nerve. Despite reliable sciatic nerve block, the fading effect of the single shot saphenous nerve block might have caused pain at the medial aspect to the ankle and foot and consequently might have led to elevated opioid consumption on POD 1. Thus, we could not distinguish, if 
opioid request was referred to poor catheter performance or terminated saphenous nerve block. However, the number of saphenous nerve blocks applied was not different between both catheter groups. Overall, the amount of opioid consumption in the present study is consistent with previous studies investigating continuous sciatic nerve block for foot and ankle surgery [25, 30-32].

\section{Additional anesthesia procedures}

A further aspect of the discussion is the possible influence of additional anesthetic procedures on postoperative pain intensity. Most patients $(80.7 \%$ ) underwent adjunct general anesthesia, whereas 11.4 $\%$ received additional spinal anesthesia to the distal sciatic block. Sedation or additional peripheral regional anesthesia was given to the remaining $7.9 \%$. The choice of procedure was individually adapted to the comorbidities and patients request. Due to randomisation and group size there were no significant differences regarding the distribution between of additional anesthetic procedures between both groups, hence a possible influence might be negligible in this investigation. The question of whether spinal anesthesia has an influence on the postoperative pain level has not been conclusively clarified until now. YaDeau et al. compared general and spinal anesthesia, each in combination with PNB for operations on the ankle and foot in a recent randomised controlled trial. A significant difference of pain scores in favour of spinal anesthesia was found only one hour after the end of the operation [34]. In contrast to long acting morphine we used fentanyl as intrathecal supplemental opioid for spinal anesthesia. Thus, any effect on pain intensity beyond first 12 hours seems unlikely.

\section{Catheter orifices}

We compared in the present study a self-coiling catheter with a closed tip and six lateral microholes with a regular straight catheter that has only a single orifice at the end. One might question if this could have influenced our results. Fredrickson et al. investigated the outflow of injected fluid on catheters with a different number of orifices. They showed a dependency of the fluid spread pattern on the fluid flow rate. Below $80 \mathrm{ml}$ per hour, fluid left multi-orifice catheters only on the most proximal orifice [35]. Only an injection rate over $100 \mathrm{ml}$ per hour delivered all microholes. Considering our study's flowrate of local anesthetics of 6-10 ml per hour, the self-coiling catheter likely functioned rather as a single orifice catheter. Thus, we do not expect any relevant advantage of the multiple catheter orifice configuration in the present study. Moreover, considering that the SCC had been positioned only $2.4 \mathrm{~cm}$ into the target space within the perineural facial sheath, with continuous infusion the local anesthetic may have left the most proximal orifice only missing the target space partially if the catheter gets retracted by muscle movements. Clinical data regarding the influence of catheter orifice design on quality of pain management is conflicting, despite LA bolus application was used. Consequently, it can be concluded that catheters with multiple openings, such as the SCC, also function like conventional end-hole catheters at clinically relevant infusion rates. In this study there was a maximum continuous flow rate of $10 \mathrm{ml}$ per hour. Only bolus applications by the acute pain service were probably applicated at speeds above $100 \mathrm{ml}$ per hour. Since this injection was performed manually from a $10 \mathrm{ml}$ syringe, no more precise statements can be made here about the application speed [36,37]

\section{Dislocation rate}


Despite the widespread use of continuous regional anesthesia the topic of perineural catheter dislocation is not well elucidated, neither in studies nor in clinical practice. Thus, the results of our study provide new insights regarding factors contributing to catheter dislocation. To our best knowledge, there is no clinical study investigating dislocation rate of self-coiling catheters compared to regular catheters so far. Luyet et al had shown a decreased initial misplacement rate for self-coiling catheters in human cadavers. However, the cadaver study design did not address dislocation rates in the further course [19].

Significantly fewer self-coiling catheters (14\%) slipped out of the subparaneural target space during the study period than regular catheters with straight ends (60\%). This significant difference might be caused by different insertion distances within the perineural fascia sheath. Ilfeld et al. [38] and Steffel et al. [16] described a higher dislodgement rate for lower insertion distance at the nerve. We used a regular straight catheter with an indwelling metal wire in the control group. Such firm catheters often pass the target structure and protrude out of the perineural fascia sheath during initial insertion of around $3 \mathrm{~cm}$ beyond the needle tip, especially if catheters are advanced in-plane perpendicular to the SAX imaged nerve. Thus, we had to adjust the catheter by retraction until LA injection was distributed well within the perineural fascia sheath under sonographic view. The self-coiling catheter provide a more reliable initial placement without passing the target space if the insertion distance of $3 \mathrm{~cm}$ beyond the needle tip is not exceeded using an in-plane approach [19]. Thus self-coiling catheters require less likely a withdrawal due to initial misplacement and have a longer catheter segment remaining around the target structure.

For postoperative evaluation of catheter position and initial confirmation of correct catheter placement we used sonographic imaging of saline bolus via the catheter. Although the efficacy of continuous regional anesthesia is depending on proper catheter location, assessment of catheter position is still not a common procedure neither in studies nor in clinical routine [39]. Postoperative break through pain and need of additional systemic analgesic medication are often considered surrogate markers for insufficient catheter performance due to any reason. However, this concept is misleading to prove an incorrect catheter position. It has to be considered that the visualization of catheter position is often compromised by sterile dressing and swollen tissue in the affected area.

Several methods for visualization of catheter position have been described in the literature, a.e. imaging of injected fluid (either saline, LA, or contrast medium) or air spreading out of catheter orifice or direct visualization of the catheter [14, 16, 40-42]. Imaging techniques include high resolution ultrasound (HRUS), computer tomography (CT), or magnetic resonance imaging (MRI) [19, 40, 41, 43]. There are pros and cons of each technique that we would like to discuss briefly. We decided for the injection of saline to prove catheter tip position because it is a safe and straightforward method that is part of our daily clinical routine. Moreover, it avoids unnecessary local anesthetic doses and harm to vulnerable structures nearby. The use of air or agitated fluid with microbubbles may enhance contrast and visualization of the injectate. However, spreading air within the tissue decreases markedly sonographic imaging quality of the target structure and surrounding tissue by scattered ultrasound waves. In contrast, visualization of saline via the catheter is similar to common procedure of observing local anesthetic injection via the cannula. Finally, direct visualization of the catheter is less favourable. On the one hand, despite the improved 
echogenity of catheters they hardly alignm with the ultrasound beam plane like a firm needle. This issue is aggravated by the use of self-coiling catheters. On the other hand, according similar to the injection over needles, control of spread of LA around the target structure is more important for a reliable block success than the catheter tip position itself. Compared to CT and MRI high resolution ultrasound imaging is commonly available. HRUS is a real point of care technique that can be applied as often as desired by the anesthesiologist avoiding unnecessary patient transports and staff expenses. Furthermore, CT examination exposes patients to radiation burden.

In our study the most dislocations were discovered after arrival at the PACU. It can be assumed that passive and/or active movement of the thigh musculature occurs when the patient is positioned prior to surgery or during patient transfer to bed. This causes mechanical traction to the catheter within the biceps femoris muscle pulling the catheter back outward of the target space within the nerve-surrounding fascia.

The dislocation rate within tissue was $14 \%$ for the self-coiling catheter or $60 \%$ for the regular straight catheter. Marhofer et al. examined the dislocation rates within the tissue in healthy volunteers. They determined a dislocation rate after movement of $25 \%$ and $5 \%$ for perineural femoral and interscalene catheter [15]. In contrast to our investigation, catheters were only examined for six hours after insertion. In addition, both catheters were placed using the out-of-plane technique, which is more robust against dislocation [43].

Only two studies address internal dislocation rates for continuous popliteal sciatic nerve block. Steffel et al. compared a catheter-over-needle (CON) with a conventional catheter-through-needle (CTN) technique in human cadaver [16]. $27 \%$ of the CON catheters dislocated from the fascial sheath of the sciatic nerve. In contrast, all conventional catheters remained perineural. Remarkably, Steffel et al. did not evaluate the spread of the local anesthetic, but only visualized sonographically the suspected end of the catheter in the sonography. However, LA spread around the target is the decisive determinant for a sufficient nerve block. Comparisons with our study are difficult, since only a tiny cohort of 30 persons was involved and the tissue characteristics of the body donors cannot be considered identical to those of living subjects [16]. In contrast to our patients, only passive flexion movements were performed on the body donors. However, we believe that an active contraction and relaxing of the muscle contributes considerably to the movements of the catheter within the tissue. Hauritz and colleagues compared two different approaches for popliteal sciatic blockade, regarding dislocation within the tissue. They confirmed catheter location 48 $\mathrm{h}$ after application by means of an MRI contrast bolus [10]. Whereas a dislocation rate of $10 \%$ for the out-of-plane approach was reported, the use of an in-plane technique similar to our study protocol resulted in a much higher dislocation rate of $40 \%$. We found an even higher dislocation rate of $60 \%$ for conventional catheters in our study. Important difference is the longer part of catheter remaining under the perineural fascia sheath. A catheter distance within the perineural fascia sheath of $1.6 \mathrm{~cm}$ in our study compared to $3.4 \mathrm{~cm}$ in the investigation of Hauritz et al. may increase catheter dislocations due to traction forces as already mentioned before. Interestingly, the application of self-coiling catheter using the in-plane approach decreased the dislocation rate to a level reported by Hauritz et al. for the out-of-plane 
technique. Whereas regular straight perineural catheters seem more reliable in point of dislocation rate using out-of-plane approach, whereas the self-coiling catheters may be placed with good results performing the in-plane technique.

\section{Leakage and catheter shift at the insertion site}

The maximum leakage rate was $31.4 \%$ in both groups in our study. This is comparable with the leaking rate of $31 \%$ for CTN technique in another study [40]. Lower leakage rates of $13.9 \%$ for continuous distal sciatic catheters were reported [44]. Leakage problems commonly occur with catheter-through-needle approaches, because needle's diameter is larger than the catheter. Thus, the tissue is not sealing the puncture track along the catheter and injected fluid as well as interstitial fluid or blood can flow retrogradely. Though catheter-over-needle technique decreases the occurrence of leaking, no superiority regarding dislocation rates has been shown in clinical trials so far $[40,45,46]$. Accordingly, we did not observe any relation between leakage and dislocations since both study groups showed the same leakage rate. Dislocation rates in terms of the catheter slipping out of the skin at the insertion site are reported with an incidence of 0.5 to $26 \%[8,9]$. In our study we considered an outward slipping at the insertion site of $1 \mathrm{~cm}$ or more as a clinically relevant movement. This was evident in $10 \%$ in SCC group and $12.6 \%$ in RSC group. According to findings of Marhofer et al. we could not observe a significant impact of outwardly slipped catheters at insertion site on the dislocation rate at target area [15].

\section{Complications}

The overall complication rate was very low. No persistent neurologic deficit was observed. The most severe complication was a patient with a metallic taste several hours after catheter application considered as a mild sign of local anesthetic systemic toxicity. Ropivacaine infusion was stopped. Neither a negative aspiration test nor sonographic imaging of fluid spread via the catheter revealed a secondary intravascular dislocation. The catheter was removed immediately, followed by complete regression of symptoms. The overall mild infection rate of $5.7 \%$ observed here ranges within the results of other studies $[47,48]$. Mild infection was considered as any redness of the puncture site during daily visit. The catheters were removed immediately without any further sequels.

In this study, catheter occlusion occurred in one patient from each cohort (total $1.4 \%$ ). Ma et al. reported this rare incident with a rate of $1 \%$ [44].

\section{Limitations}

Our results are limited to the use of ropivacaine for the initial bolus. The dislocated catheters probably would have been earlier discovered by using a short- or middle-long acting local anesthetic for the initial bolus. Additionally, for rescue pain management patients received opioids orally by nurses only on demand in a fixed dosage of $10 \mathrm{mg}$ Oxycodone. A more finely tuned opioid application, e.g. by patient controlled intravenous analgesia could have led to a clearer reflection of the actual need for additional painkillers. 
Another limitation is the single shot concept for saphenous nerve block with limited pain relief of 12- 18 hours. Thus, NRS scores may have been influenced by pain perception in saphenous nerve innervation area.

Furthermore, the design of our study was not double blinded since the ultrasound examiner occasionally could have drawn conclusions regarding catheter type by watching insertion depth specific catheter length graduations.

The results of this study apply only to our setting of short axis view of the nerve and in-plane needle approach. Beyond that, our results are not applicable for other catheter designs or alternative insertion sites.

In the case of sonographically confirmed catheter dislocation in situ, no further positional checks were performed. Whether it is possible that the misalignment could spontaneously convert to a renewed perineural position or not remains unclear.

Furthermore, extrafascial dislocation does not necessarily mean a complete loss of effectiveness. Our continuous ropivacaine dose may have been still sufficient to release pain by local anesthetic spreading toward the nerve along the residual puncture pathway or by diffusion through the connective tissue. Experience from the efficacy of interfascial plane blocks (e.g. the erector spinae block), we suspect some analgetic effects of even low amounts of LA by blocking small C-fibres, even if the application site is not close to the target nerve.

\section{Conclusions}

The self-coiling catheter design for continuous popliteal sciatic nerve block offers a superior postoperative pain control and a lower dislocation rate within the tissue than a conventional straight catheter design. Secondary migration, away from the nerve target structure, could only be visualised sonographically and was not related to the external appearance at the puncture site. Additional studies for other alternative techniques and localization are warranted to further evaluate this design.

\section{Abbrevations}

AE: adverse event; CTN: Catheter-through-needle; CON: Catheter-over-needle; CONSORT: Consolidated Standards of Reporting Trials; IP: in-plane; LA: local anesthetic; NRS: numeric rating scale; OOP: out-ofplane; PACU: post anesthesia care unit; PNB: peripheral nerve block; POD: postoperative day; RA: regional anesthesia; RSC: regular straight catheter; SAE: severe adverse event; SAX: short axis view; SCC: selfcoiling catheter; US: ultrasound

\section{Declarations}

\section{Ethics approval and consent to participate}


The present trial was approved by the local institutional review board of the TU Dresden, the "Ethikkomission an der Technischen Universität Dresden", Dresden, Germany (approval number: EK 150042016). Written informed consent was obtained from all participants with the informed consent form prior to trial enrolment. All methods were carried out in accordance with the Declaration of Helsinki.

\section{Consent for publication}

Not applicable.

\section{Availability of data and materials}

The datasets used and/or analysed during the current study are available from the corresponding author on reasonable request.

\section{Competing interests}

OV performs consulting services and receives lecture honoraria from Pajunk, Geisingen, Germany and Sintetica $\mathrm{GmbH}$, Muenster, Germany. All other authors declare that they have no competing interests.

\section{Funding}

The present trial was supported by Pajunk, Geisingen, Germany, who provided the trial catheters, but had no part in study design, data collection, interpreting the results or preparing the manuscript.

Support was provided by the Open Access Funding by the Publication Fund of the TU Dresden.

\section{Author's contribution}

OV and RN designed the study protocol, generated the random allocation sequence, enrolled participants, and collected data and contributed to manuscript preparation. OV, AO and TM helped with data collection including performance of sonographic controls of the catheter positions. RN, KS and TR performed the statistical data analysis. TR, OV and TK helped preparing the manuscript and interpreting the data. All authors have read and approved the manuscript.

\section{Acknowledgements}

The authors thank the anesthetic and surgical staff of the University Center of Orthopaedics and Traumatology and Comprehensive Pain Center, University Hospital Carl Gustav Carus Dresden, Dresden, Germany.

Data was part of thesis by Rosa Nickl.

\section{Author's information}


${ }^{1}$ Department of Anesthesiology and Critical Care Medicine, University Hospital Carl Gustav Carus at the Technische Universität Dresden, Fetscherstr. 74, 01307 Dresden, Germany.

2 Institute for Medical Informatics and Biometry, Medical Faculty Carl Gustav Carus, Technische Universität Dresden, Fetscherstr. 74, 01307 Dresden, Germany.

\section{References}

1. Chelly JE, Ghisi D, Fanelli A. Continuous peripheral nerve blocks in acute pain management. British Journal of Anaesthesia. 2010;105:i86-96.

2. Gerbershagen HJ, Aduckathil S, van Wijck AJM, Peelen LM, Kalkman CJ, Meissner W. Pain Intensity on the First Day after Surgery: A Prospective Cohort Study Comparing 179 Surgical Procedures. Anesthesiology. 2013;118:934-44.

3. Salviz EA, Xu D, Frulla A, Kwofie K, Shastri U, Chen J, et al. Continuous Interscalene Block in Patients Having Outpatient Rotator Cuff Repair Surgery: A Prospective Randomized Trial. Anesthesia \& Analgesia. 2013;117:1485-92.

4. Peng L, Ren L, Qin P, Chen J, Feng P, Lin H, et al. Continuous Femoral Nerve Block versus Intravenous Patient Controlled Analgesia for Knee Mobility and Long-Term Pain in Patients Receiving Total Knee Replacement: A Randomized Controlled Trial. Evidence-Based Complementary and Alternative Medicine. 2014;2014:1-12.

5. Sakai N, Inoue T, Kunugiza Y, Tomita T, Mashimo T. Continuous Femoral Versus Epidural Block for Attainment Of $120^{\circ}$ Knee Flexion After Total Knee Arthroplasty: A Randomized Controlled Trial. The Journal of Arthroplasty. 2013;28:807-14.

6. Albrecht E, Bathory I, Fournier N, Jacot-Guillarmod A, Farron A, Brull R. Reduced hemidiaphragmatic paresis with extrafascial compared with conventional intrafascial tip placement for continuous interscalene brachial plexus block: a randomized, controlled, double-blind trial. British Journal of Anaesthesia. 2017;118:586-92.

7. Abildgaard JT, Lonergan KT, Tolan SJ, Kissenberth MJ, Hawkins RJ, Washburn R, et al. Liposomal bupivacaine versus indwelling interscalene nerve block for postoperative pain control in shoulder arthroplasty: a prospective randomized controlled trial. Journal of Shoulder and Elbow Surgery. 2017;26:1175-81.

8. Ahsan ZS, Carvalho B, Yao J. Incidence of Failure of Continuous Peripheral Nerve Catheters for Postoperative Analgesia in Upper Extremity Surgery. The Journal of Hand Surgery. 2014;39:324-9.

9. Gurnaney H, Kraemer FW, Maxwell L, Muhly WT, Schleelein L, Ganesh A. Ambulatory Continuous Peripheral Nerve Blocks in Children and Adolescents: A Longitudinal 8-Year Single Center Study. Anesthesia \& Analgesia. 2014;118:621-7.

10. Hauritz RW, Pedersen EM, Linde FS, Kibak K, Børglum J, Bjoern S, et al. Displacement of popliteal sciatic nerve catheters after major foot and ankle surgery: a randomized controlled double-blinded magnetic resonance imaging study. British Journal of Anaesthesia. 2016;117:220-7. 
11. Fredrickson MJ, Leightley P, Wong A, Chaddock M, Abeysekera A, Frampton C. An analysis of 1505 consecutive patients receiving continuous interscalene analgesia at home: a multicentre prospective safety study. Anaesthesia. 2016;71:373-9.

12. Walker BJ, Long JB, De Oliveira GS, Szmuk P, Setiawan C, Polaner DM, et al. Peripheral nerve catheters in children: an analysis of safety and practice patterns from the pediatric regional anesthesia network (PRAN). British Journal of Anaesthesia. 2015;115:457-62.

13. Edward Kim T, Howard SK, Funck N, Kyle Harrison T, Walters TL, Wagner MJ, et al. A randomized comparison of long-axis and short-axis imaging for in-plane ultrasound-guided popliteal-sciatic perineural catheter insertion. J Anesth. 2014;28:854-60.

14. Swenson JD, Davis JJ, DeCou JA. A Novel Approach for Assessing Catheter Position After Ultrasound-Guided Placement of Continuous Interscalene Block: Anesthesia \& Analgesia. 2008;106:1015-6.

15. Marhofer D, Marhofer P, Triffterer L, Leonhardt M, Weber M, Zeitlinger M. Dislocation rates of perineural catheters: a volunteer study. British Journal of Anaesthesia. 2013;111:800-6.

16. Steffel L, Howard SK, Borg L, Mariano ER, Leng JC, Kim TE. Randomized comparison of poplitealsciatic perineural catheter tip migration and dislocation in a cadaver model using two catheter designs. Korean Journal of Anesthesiology. 2017;70-72.

17. Ilfeld BM, Fredrickson MJ, Mariano ER. Ultrasound-Guided Perineural Catheter Insertion: Three Approaches but Few Illuminating Data. Regional Anesthesia and Pain Medicine. 2010;35:123-6.

18. Luyet C, Meyer C, Herrmann G, Hatch GM, Ross S, Eichenberger U. Placement of coiled catheters into the paravertebral space*: Placement of coiled catheters in the paravertebral space. Anaesthesia. 2012;67:250-5.

19. Luyet C, Seiler R, Herrmann G, Hatch GM, Ross S, Eichenberger U. Newly Designed, Self-Coiling Catheters for Regional Anesthesia-An Imaging Study: Regional Anesthesia and Pain Medicine. 2011;36:171-6.

20. Eldridge SM, Campbell MJ, Bond CM, Hopewell S, Thabane L, Lancaster GA. CONSORT 2010 statement: extension to randomised pilot and feasibility trials. Pilot Feasibility Stud. 2016;2:64, s40814-016-0105-8.

21. Andersen HL, Andersen SL, Tranum-Jensen J. Injection Inside the Paraneural Sheath of the Sciatic Nerve: Direct Comparison Among Ultrasound Imaging, Macroscopic Anatomy, and Histologic Analysis. Regional Anesthesia and Pain Medicine. 2012;37:410-4.

22. Christiansen CB, Madsen MH, Rothe $C$, Andreasen AM, Lundstrøm LH, Lange KHW. Volume of ropivacaine $0.2 \%$ and sciatic nerve block duration: $A$ randomized, blinded trial in healthy volunteers. Acta Anaesthesiol Scand. 2020;64:238-44.

23. Ding DY, Manoli A, Galos DK, Jain S, Tejwani NC. Continuous Popliteal Sciatic Nerve Block Versus Single Injection Nerve Block for Ankle Fracture Surgery: A Prospective Randomized Comparative Trial. Journal of Orthopaedic Trauma. 2015;29:393-8. 
24. Zaric D, Boysen K, Christiansen J, Haastrup U, Kofoed H, Rawal N. Continuous popliteal sciatic nerve block for outpatient foot surgery - a randomized, controlled trial. Acta Anaesthesiol Scand. 2004;48:337-41.

25. Elliot R, Pearce CJ, Seifert C, Calder DJ. Continuous Infusion Versus Single Bolus Popliteal Block Following Major Ankle and Hindfoot Surgery: A Prospective, Randomized Trial. Foot \& Ankle International. 2010;31:1043-7.

26. Kåsine T, Romundstad L, Rosseland LA, Fagerland MW, Kessler P, Omenås IN, et al. Ultrasonographic needle tip tracking for in-plane infraclavicular brachialis plexus blocks: a randomized controlled volunteer study. Reg Anesth Pain Med. 2020;45:634-9.

27. Fisker AK, Iversen BN, Christensen S, Linde F, Nielsen KK, Børglum J, et al. Combined saphenous and sciatic catheters for analgesia after major ankle surgery: a double-blinded randomized controlled trial. Can J Anesth/J Can Anesth. 2015;62:875-82.

28. Jarrell K, McDonald E, Shakked R, Nicholson K, Kasper V, Raikin SM. Combined Popliteal Catheter With Single-Injection vs Continuous-Infusion Saphenous Nerve Block for Foot and Ankle Surgery. Foot Ankle Int. 2018;39:332-7.

29. Bjørn S, Linde F, Nielsen KK, Børglum J, Hauritz RW, Bendtsen TF. Effect of Perineural Dexamethasone on the Duration of Single Injection Saphenous Nerve Block for Analgesia After Major Ankle Surgery: A Randomized, Controlled Study. Regional Anesthesia and Pain Medicine. 2017;42:210-6.

30. Bendtsen TF, Nielsen TD, Rohde CV, Kibak K, Linde F. Ultrasound Guidance Improves a Continuous Popliteal Sciatic Nerve Block When Compared With Nerve Stimulation: Regional Anesthesia and Pain Medicine. 2011;36:181-4.

31. Capdevila X, Dadure C, Bringuier S, Bernard N, Biboulet P, Gaertner E, et al. Effect of Patient-controlled Perineural Analgesia on Rehabilitation and Pain after Ambulatory Orthopedic Surgery: A Multicenter Randomized Trial. Anesthesiology. 2006;105:566-73.

32. Ilfeld BM, Morey TE, Wang RD, Enneking FK. Continuous Popliteal Sciatic Nerve Block for Postoperative Pain Control at Home: A Randomized, Double-Blinded, Placebo-Controlled Study. Anesthesiology. 2002;97:959-65.

33. White PF, Issioui T, Skrivanek GD, Early JS, Wakefield C. The Use of a Continuous Popliteal Sciatic Nerve Block After Surgery Involving the Foot and Ankle: Does It Improve the Quality of Recovery?: Anesthesia \& Analgesia. 2003;97:1303-9.

34. YaDeau JT, Fields KG, Kahn RL, LaSala VR, Ellis SJ, Levine DS, et al. Readiness for Discharge After Foot and Ankle Surgery Using Peripheral Nerve Blocks: A Randomized Controlled Trial Comparing Spinal and General Anesthesia as Supplements to Nerve Blocks. Anesthesia \& Analgesia. 2018;127:759-66.

35. Fredrickson MJ. Randomised Comparison of an End-Hole, Triple-Hole and Novel Six-Hole Catheter for Continuous Interscalene Analgesia. Anaesthesia and Intensive Care. 2014;42:37-42. 
36. Novello-Siegenthaler A, Hamdani M, Iselin-Chaves I, Fournier R. Ultrasound-guided continuous femoral nerve block: a randomized trial on the influence of femoral nerve catheter orifice configuration (six-hole versus end-hole) on post-operative analgesia after total knee arthroplasty. BMC Anesthesiol. 2018;18:191.

37. Fredrickson MJ, Ball CM, Dalgleish AJ. Catheter Orifice Configuration Influences the Effectiveness of Continuous Peripheral Nerve Blockade: Regional Anesthesia and Pain Medicine. 2011;36:470-5.

38. Ilfeld BM, Sandhu NS, Loland VJ, Madison SJ, Suresh PJ, Mariano ER, et al. Ultrasound-Guided (Needle-in-Plane) Perineural Catheter Insertion: The Effect of Catheter-Insertion Distance on Postoperative Analgesia. Regional Anesthesia and Pain Medicine. 2011;36:261-5.

39. Schnabel A, Meyer-Frießem CH, Zahn PK, Pogatzki-Zahn EM. Ultrasound compared with nerve stimulation guidance for peripheral nerve catheter placement: a meta-analysis of randomized controlled trials. British Journal of Anaesthesia. 2013;111:564-72.

40. Finneran JJ, Swisher MW, Gabriel RA, Said ET, Abanobi MU, Abramson WB, et al. Suture-method versus Through-the-needle Catheters for Continuous Popliteal-sciatic Nerve Blocks. Anesthesiology. 2020;132:854-66.

41. Marhofer $P$, Anderl W, Heuberer P, Fritz M, Kimberger O, Marhofer D, et al. A retrospective analysis of 509 consecutive interscalene catheter insertions for ambulatory surgery. Anaesthesia. 2015;70:41-6.

42. Brookes J, Sondekoppam R, Armstrong K, Uppal V, Dhir S, Terlecki M, et al. Comparative evaluation of the visibility and block characteristics of a stimulating needle and catheter vs an echogenic needle and catheter for sciatic nerve block with a low-frequency ultrasound probe. British Journal of Anaesthesia. 2015;115:912-9.

43. Hauritz RW, Pedersen EM, Linde FS, Kibak K, Børglum J, Bjoern S, et al. Displacement of popliteal sciatic nerve catheters after major foot and ankle surgery: a randomized controlled double-blinded magnetic resonance imaging study. British Journal of Anaesthesia. 2016;117:220-7.

44. Ma H-H, Chou T-FA, Tsai S-W, Chen C-F, Wu P-K, Chen W-M. The efficacy and safety of continuous versus single-injection popliteal sciatic nerve block in outpatient foot and ankle surgery: a systematic review and meta-analysis. BMC Musculoskelet Disord. 2019;20:441.

45. Ip VHY, Rockley MC, Tsui BCH. The catheter-over-needle assembly offers greater stability and less leakage compared with the traditional counterpart in continuous interscalene nerve blocks: a randomized patient-blinded study. Canadian Journal of Anesthesia/Journal canadien d'anesthésie. 2013;60:1272-3.

46. Tsui BC, Ip VH. Catheter-over-needle method reduces risk of perineural catheter dislocation. British Journal of Anaesthesia. 2014;112:759-60.

47. Morin AM, Kerwat KM, Klotz M, Niestolik R, Ruf VE, Wulf $H$, et al. Risk factors for bacterial catheter colonization in regional anaesthesia. BMC Anesthesiology. 2005;5:1.

48. Neuburger M, Breitbarth J, Reisig F, Lang D, Büttner J. Komplikationen bei peripherer Katheterregionalanästhesie: Untersuchungsergebnisse anhand von 3491 Kathetern. Der Anaesthesist. 2006;55:33-40. 
49. Kerwat K, Eberhart L, Kerwat M, Hörth D, Wulf H, Steinfeldt T, et al. Chlorhexidine Gluconate Dressings Reduce Bacterial Colonization Rates in Epidural and Peripheral Regional Catheters. BioMed Research International. 2015;2015:149785.

50. Aubuchon A, Arnold WD, Bracewell A, Hoyle JC. Sciatic neuropathy due to popliteal fossa nerve block: Short Report. Muscle \& Nerve. 2017;56:822-4.

51. Park YU, Cho JH, Lee DH, Choi WS, Lee HD, Kim KS. Complications After Multiple-Site Peripheral Nerve Blocks for Foot and Ankle Surgery Compared With Popliteal Sciatic Nerve Block Alone. Foot \& Ankle International. 2018;39:731-5.

52. Uchino T, Miura M, Oyama Y, Matsumoto S, Shingu C, Kitano T. Lateral deviation of four types of epidural catheters from the lumbar epidural space into the intervertebral foramen. J Anesth. 2016;30:583-90.

53. Mariano ER, Yun RDH, Kim TE, Carvalho B. Application of Echogenic Technology for Catheters Used in Ultrasound-Guided Continuous Peripheral Nerve Blocks. Journal of Ultrasound in Medicine. 2014;33:905-11.

54. Takatani J, Takeshima N, Okuda K, Uchino T, Noguchi T. Ultrasound visibility of regional anesthesia catheters: an in vitro study. Korean J Anesthesiol. 2012;63:59-64.

55. Moy DM, Kim TE, Harrison TK, Leng JC, Carvalho B, Howard SK, et al. Comparative Echogenicity of an Epidural Catheter and 2 New Catheters Designed for Ultrasound-Guided Continuous Peripheral Nerve Blocks: Echogenicity of 3 Regional Anesthesia Catheters. J Ultrasound Med. 2017;36:2571-6.

56. Jaime F, Mandell GL, Vallejo MC, Ramanathan S. Uniport soft-tip, open-ended catheters versus multiport firm-tipped close-ended catheters for epidural labor analgesia: a quality assurance study. Journal of Clinical Anesthesia. 2000;12:89-93.

57. Shih C-K, Wang F-Y, Shieh C-F, Huang J-M, Lu I-C, Wu L-C, et al. Soft catheters reduce the risk of intravascular cannulation during epidural block-A retrospective analysis of 1117 cases in a medical center. The Kaohsiung Journal of Medical Sciences. 2012;28:373-6.

\section{Figures}

\section{Figure 1}

Study catheters and needle. a+c Self-Coiling Catheter (Sono Long Curl Echo $20 \mathrm{G} 100$ mm, Pajunk medical products, Geising-en, Germany), a catheter tip; $c$ entire catheter $b+d$ Regular straight catheter (Sono Long Sono $20 \mathrm{G} 100$ mm, Pajunk medical products, Geising-en, Germany), b catheter tip; d entire catheter 


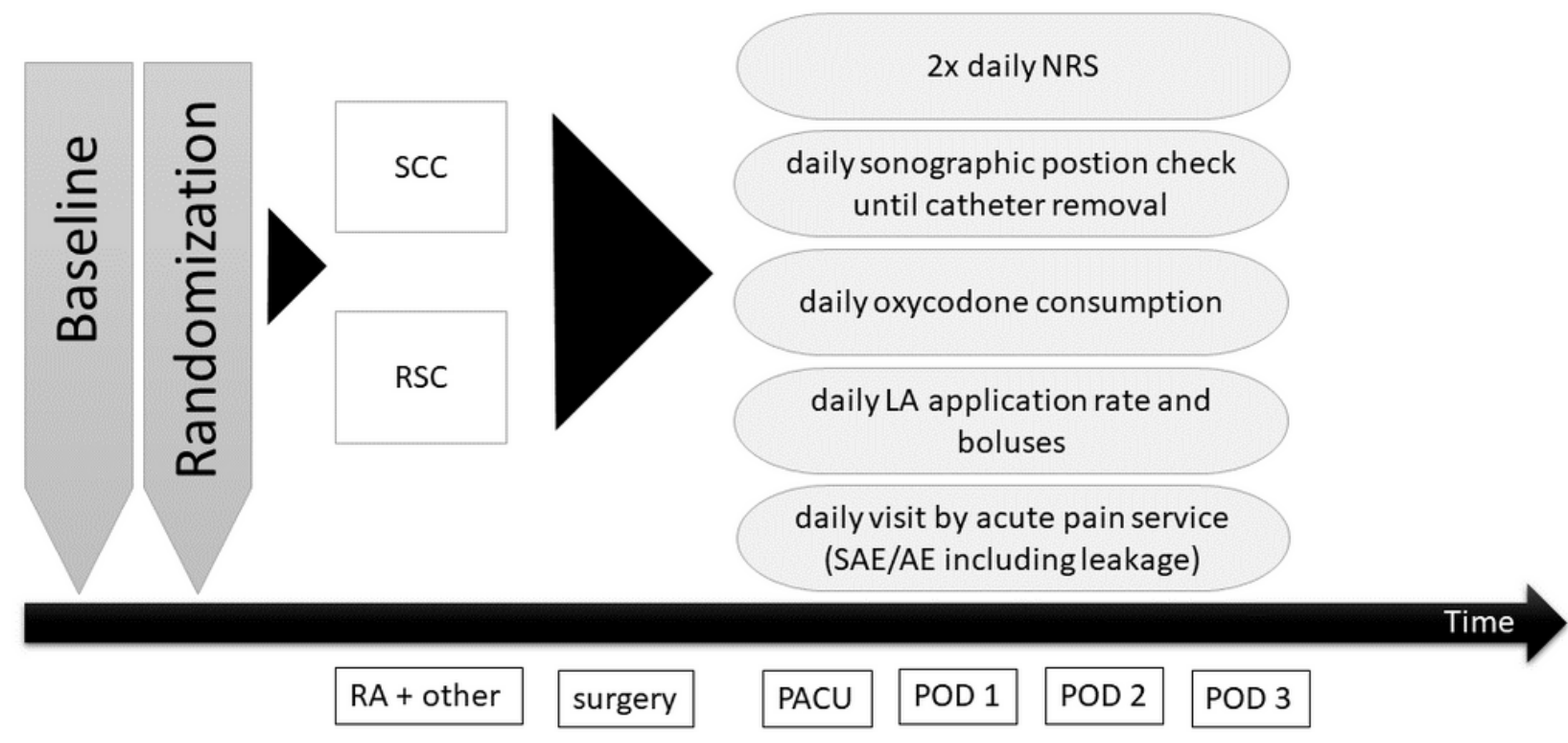

\section{Figure 2}

Time course of proceeded interventions. Baseline characteristics were assessed as shown in Table 2. AE: adverse events, NRS: numeric rating scale, PACU: postoperative anesthesia care unit, POD: postoperative day, RA: regional anesthesia, RSC: regular straight catheter group, SAE: severe ad-verse events, SCC: selfcoiling catheter group.

2

\section{Figure 3}

Catheter position categories in the sonographic image. a-perineural intrafascial position: catheter tip is located within the paraneural sheath indicated by sa-line bolus spread. b- 1 st degree dislocation, also called perineural extrafascial: catheter tip is located outside the paraneural sheath but still close to the nerve. Saline bolus (red frame line) moisture the fascial paraneural sheath (yellow frame line) but run away into surrounding tissue. This category is also recorded as dislocated for evaluation since efficacy is decreased. c- 2nd degree dislocation, catheter tip is outside the paraneural sheath without proximity to the nerve. Saline bolus spreads dif-fuse within the biceps femoris muscle. White arrow- catheter, * preferred correct position. 


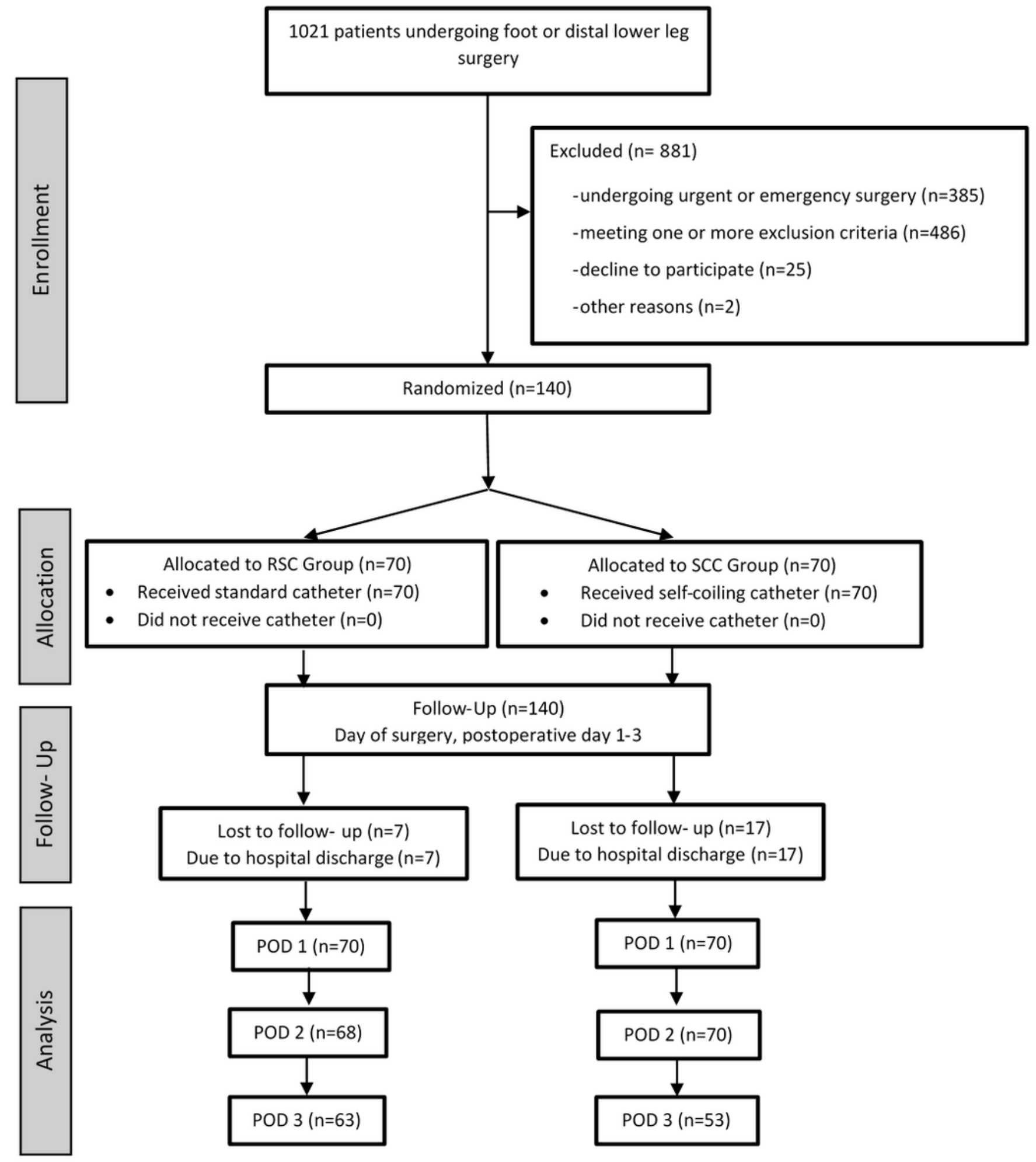

Figure 4

Flow chart. 140 consecutive patients were enrolled in this trial. Patients got lost to follow-up only because of earlier hospital discharge. No patient has withdrawn his consent. POD postoperative day, RSC regular straight catheter, SCC self-coiling catheter. 


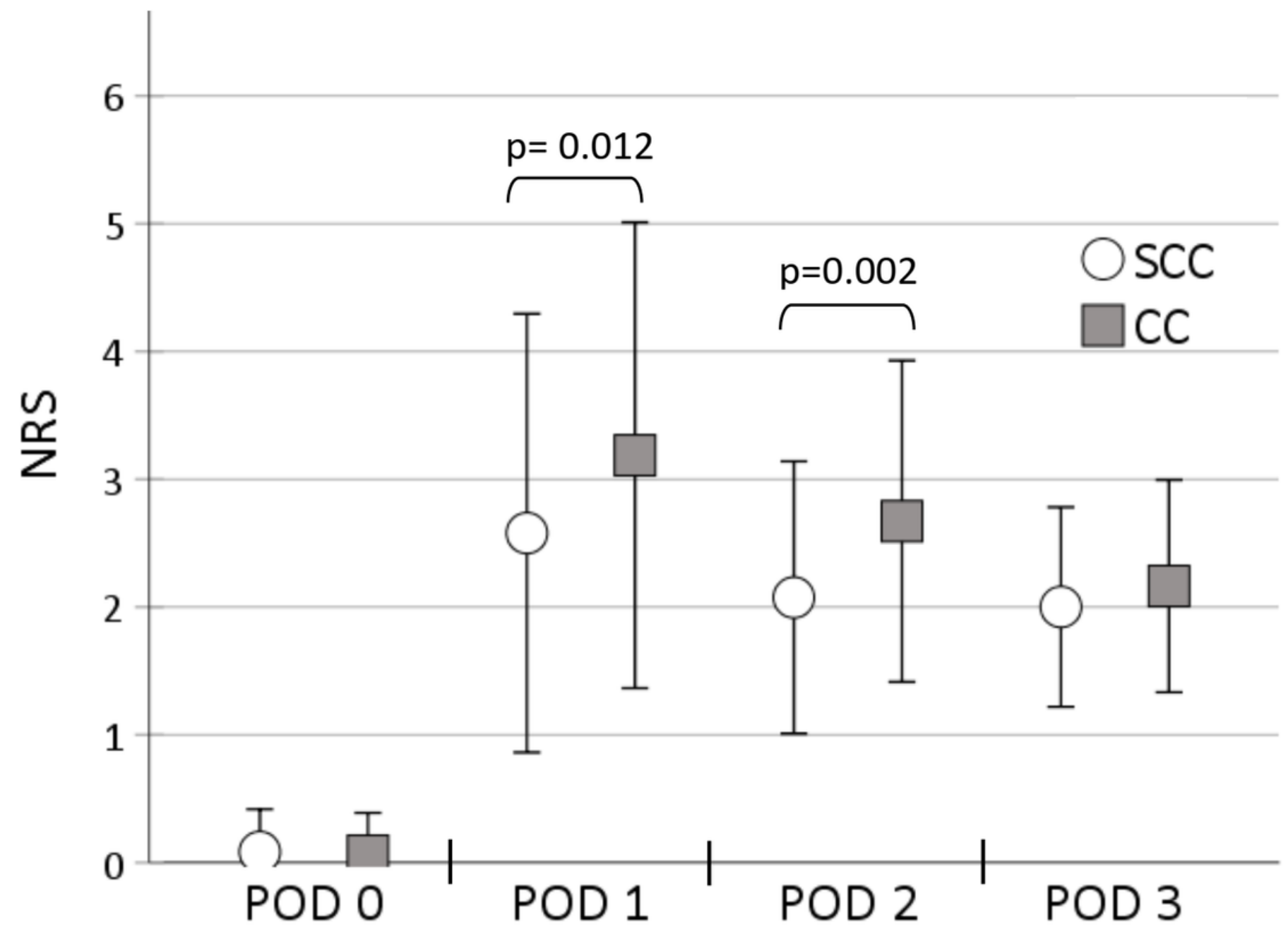

Figure 5

Postoperative pain scores. Values are given as mean \pm standard deviation on numeric rating scale. Statistical significance was considered to be at two-sided $p<0.05$. Differences between groups were analysed using Student's t-test. NRS numeric rating scale, POD postoperative day, RSC regular straight catheter, SCC self-coiling catheter. 


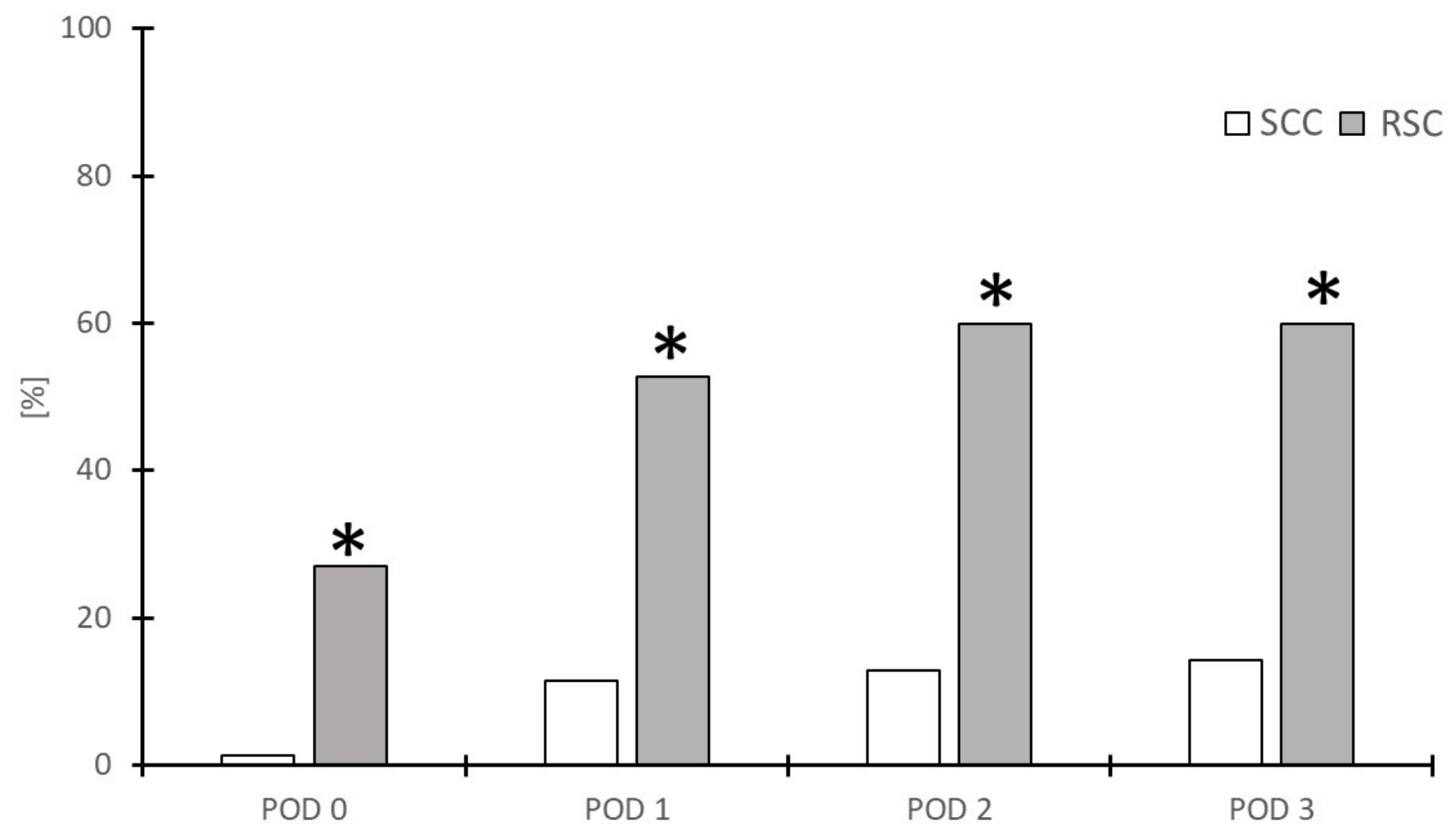

Figure 6

Percentual cumulative dislocation rate of the catheters. A Chi-square test with multiple regression approach was performed. Statistical significance was accepted at $p<0.05$. POD postoperative day, RSC regular straight catheter, SCC self-coiling catheter, ${ }^{*} p<0.05$. 


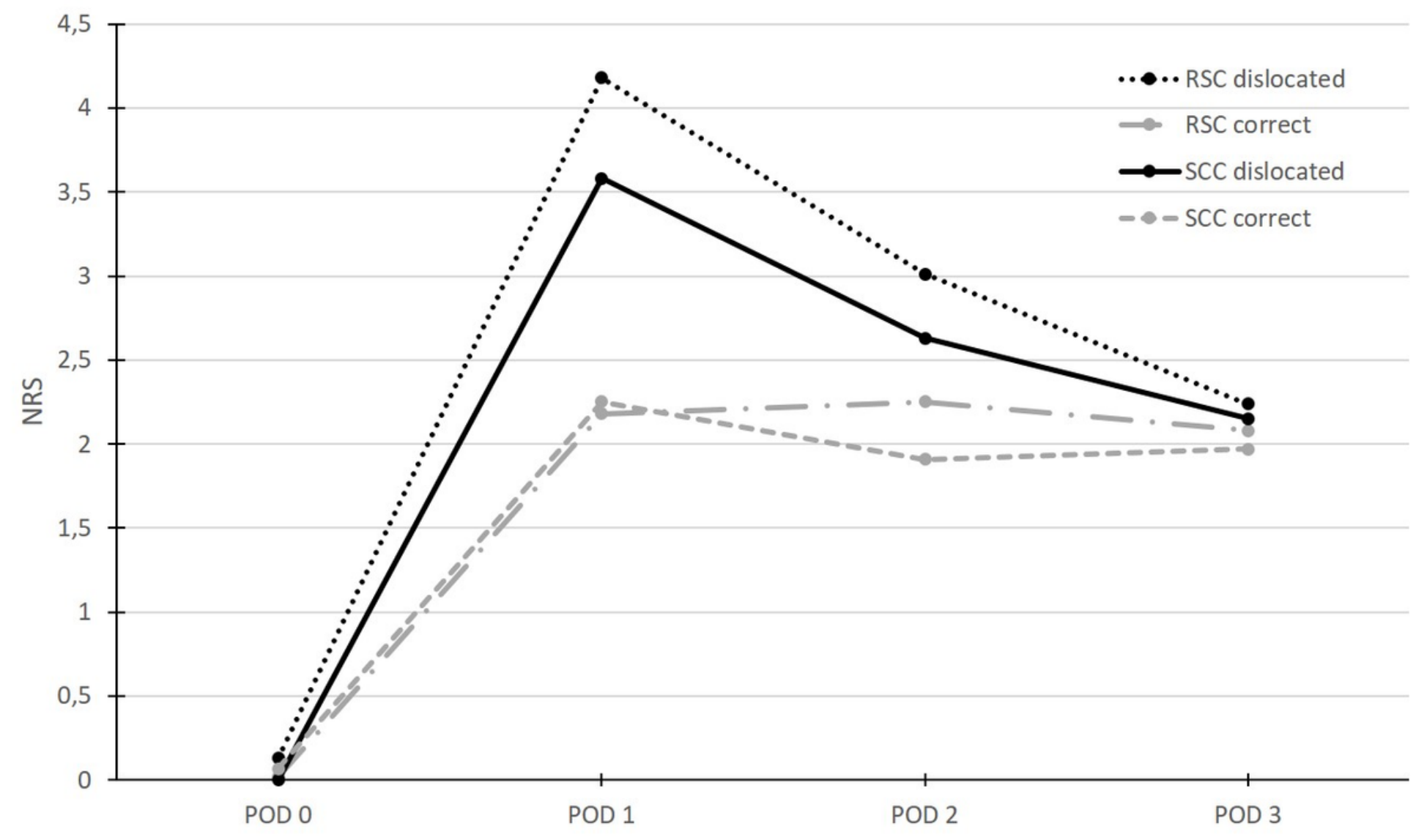

Figure 7

Mean values of NRS assessment as a function of time for the different catheter types and dislocation categories in situ. NRS numeric rating scale, POD postoperative day, RSC regular straight catheter, SCC self-coiling catheter. 